\title{
Metastatic Epithelioid Sarcoma
}

National Cancer Institute

\section{Source}

National Cancer Institute. Metastatic Epithelioid Sarcoma. NCI Thesaurus. Code C8799.

An epithelioid sarcoma which has spread to another anatomic site. 